\title{
Spin-Orbital Momentum Decomposition and Helicity Exchange in a Set of Non-Null Knotted Electromagnetic Fields
}

\author{
Manuel Arrayás ${ }^{\text {t,* }}$ and José L. Trueba ${ }^{\dagger}$ \\ Área de Electromagnetismo, Universidad Rey Juan Carlos, Calle Tulipán s/n, 28933 Móstoles (Madrid), Spain; \\ joseluis.trueba@urjc.es \\ * Correspondence: manuel.arrayas@urjc.es \\ + These authors contributed equally to this work.
}

Received: 9 March 2018; Accepted: 27 March 2018; Published: 30 March 2018

\begin{abstract}
We calculate analytically the spin-orbital decomposition of the angular momentum using completely nonparaxial fields that have a certain degree of linkage of electric and magnetic lines. The split of the angular momentum into spin-orbital components is worked out for non-null knotted electromagnetic fields. The relation between magnetic and electric helicities and spin-orbital decomposition of the angular momentum is considered. We demonstrate that even if the total angular momentum and the values of the spin and orbital momentum are the same, the behavior of the local angular momentum density is rather different. By taking cases with constant and non-constant electric and magnetic helicities, we show that the total angular momentum density presents different characteristics during time evolution.
\end{abstract}

Keywords: electromagnetic knots; helicity; spin-orbital momentum

\section{Introduction}

There has been recently some interest in the orbital-spin decomposition of the angular momentum carried by light. The total angular momentum can be decomposed into orbital and spin angular momenta for paraxial light, but for nonparaxial fields, that splitting is more controversial because their quantized forms do not satisfy the commutation relations [1,2]. For a review and references, see for example [3,4].

In this work, we provide an exact calculation of the orbital-spin decomposition of the angular momentum in a completely nonparaxial field. We compute the orbital-spin contributions to the total angular momentum analytically for a knotted class of fields [5]. These fields have nontrivial electromagnetic helicity [6,7]. We show that the existence of electromagnetic fields in a vacuum with the same constant angular momentum and orbital-spin decomposition, but different electric and magnetic helicities is possible. We find cases where the helicities are constant during the field evolution and cases where they change in time, evolving through a phenomenon of exchanging magnetic and electric components [8]. The angular momentum density presents different time evolution in each case.

The orbital-spin decomposition and its observability has been discussed in the context of the dual symmetry of Maxwell equations in a vacuum [9]. In this paper, we first make a brief review of the concept of electromagnetic duality. That duality, termed "electromagnetic democracy" [10], has been central in the work of knotted field configurations [5,11-26]. Related field configurations have also appeared in plasma physics [27-30], optics [31-35], classical field theory [36], quantum physics [37,38], various states of matter [39-43] and twistors [44,45].

We will make use of the helicity basis [7] in order to write the magnetic and electric spin of the field in that basis, which simplifies the calculations, as well as the magnetic and electric helicities' 
components. On that basis, we will get some general results, such as the difference between the magnetic and electric spin components in the Coulomb gauge is null. This conclusion coincides with the results found, for example, in [46] using a different approach. We proceed by giving the explicit calculation of the decomposition of the angular momentum into spin and orbital components for a whole class of fields, the non-null toroidal class $[5,25]$. We will show that the angular decomposition remains constant in time, while the helicities may or may not change. We provide an example of each case and plot the time evolution of the total angular momentum density. In the final section, we summarize the main results.

\section{Duality and Helicity in Maxwell Theory in a Vacuum}

In this section, we will review the definition of magnetic and electric helicities. These definitions are possible because of the dual property of electromagnetism in a vacuum. We will also describe a vector density, which can be identified with the spin density using the helicity four-current zeroth component.

Electromagnetism in a vacuum can be described in terms of two real vector fields, $\mathbf{E}$ and $\mathbf{B}$, called the electric and magnetic fields, respectively. Using the SI units, these fields satisfy Maxwell equations in a vacuum,

$$
\begin{aligned}
& \nabla \cdot \mathbf{B}=0, \quad \nabla \times \mathbf{E}+\partial_{t} \mathbf{B}=0, \\
& \nabla \cdot \mathbf{E}=0, \quad \nabla \times \mathbf{B}-\frac{1}{c^{2}} \partial_{t} \mathbf{E}=0 .
\end{aligned}
$$

Using the four-vector electromagnetic potential:

$$
A^{\mu}=\left(\frac{V}{c}, \mathbf{A}\right)
$$

where $V$ and $\mathbf{A}$ are the scalar and vector potential, respectively, the electromagnetic field tensor is:

$$
F_{\mu \nu}=\partial_{\mu} A_{\nu}-\partial_{\nu} A_{\mu}
$$

From Equation (4), the electric and magnetic field components are:

$$
\mathbf{E}_{i}=c F^{i 0}, \mathbf{B}_{i}=-\frac{1}{2} \epsilon_{i j k} F^{j k}
$$

or, in three-dimensional quantities,

$$
\mathbf{E}=-\nabla V-\frac{\partial \mathbf{A}}{\partial t}, \mathbf{B}=\nabla \times \mathbf{A}
$$

Since Equation (1) is just identities in terms of the four-vector electromagnetic potential Equation (3), by using (6), the dynamics of electromagnetism is given by Equation (2), which can be written as:

$$
\partial_{\mu} F^{\mu v}=0
$$

Partly based on the duality property of Maxwell equations in a vacuum [47], there is the idea of "electromagnetic democracy" $[9,10]$. The equations are invariant under the map $(\mathbf{E}, c \mathbf{B}) \mapsto(c \mathbf{B},-\mathbf{E})$. Electromagnetic democracy means that, in a vacuum, it is possible to define another four-potential:

$$
C^{\mu}=\left(c V^{\prime}, \mathbf{C}\right),
$$


so that the dual of the electromagnetic tensor $F_{\mu \nu}$ in Equation (4), defined as:

$$
{ }^{*} F_{\mu \nu}=\frac{1}{2} \varepsilon_{\mu \nu \alpha \beta} F^{\alpha \beta},
$$

satisfies:

$$
{ }^{*} F_{\mu v}=-\frac{1}{c}\left(\partial_{\mu} C_{\nu}-\partial_{\nu} C_{\mu}\right),
$$

or, in terms of three-dimensional fields,

$$
\mathbf{E}=\nabla \times \mathbf{C}, \quad \mathbf{B}=\nabla V^{\prime}+\frac{1}{c^{2}} \frac{\partial \mathbf{C}}{\partial t} .
$$

Equation (2) is again identities when the definitions (11) are imposed. Thus, Maxwell equations in a vacuum can be described in terms of two sets of vector potentials as in definition Equations (4) and (10), which have to satisfy the duality condition Equation (9).

In the study of topological configurations of electric and magnetic lines, an important quantity is the helicity of a vector field [48-53], which can be defined for every divergenceless three-dimensional vector field. Magnetic helicity is related to the linkage of magnetic lines. In the case of electromagnetism in a vacuum, the magnetic helicity can be defined as the integral:

$$
h_{m}=\frac{1}{2 c \mu_{0}} \int d^{3} r \mathbf{A} \cdot \mathbf{B},
$$

where $c$ is the speed of light in a vacuum and $\mu_{0}$ is the vacuum permeability. Note that, in this equation, the magnetic helicity is taken so that it has dimensions of angular momentum in SI units. Since the electric field in a vacuum is also divergenceless, an electric helicity, related to the linking number of electric lines, can also be defined as:

$$
h_{e}=\frac{\varepsilon_{0}}{2 c} \int d^{3} r \mathbf{C} \cdot \mathbf{E}=\frac{1}{2 c^{3} \mu_{0}} \int d^{3} r \mathbf{C} \cdot \mathbf{E},
$$

where $\varepsilon_{0}=1 /\left(c^{2} \mu_{0}\right)$ is the vacuum electric permittivity. Electric helicity in Equation (13) also has dimensions of angular momentum. Magnetic and electric helicities in a vacuum can be studied in terms of helicity four-currents $[6,7,9,17]$, so that the magnetic helicity density is the zeroth component of:

$$
\mathcal{H}_{m}^{\mu}=-\frac{1}{2 c \mu_{0}} A_{v}{ }^{*} F^{v \mu}
$$

and the electric helicity is the zeroth component of:

$$
\mathcal{H}_{e}^{\mu}=-\frac{1}{2 c^{2} \mu_{0}} C_{v} F^{v \mu} .
$$

The divergence of $\mathcal{H}_{m}^{\mu}$ and $\mathcal{H}_{e}^{\mu}$ is related to the time conservation of both helicities,

$$
\begin{aligned}
\partial_{\mu} \mathcal{H}_{m}^{\mu} & =\frac{1}{4 c \mu_{0}} F_{\mu v}{ }^{*} F^{\mu v}, \\
\partial_{\mu} \mathcal{H}_{e}^{\mu} & =-\frac{1}{4 c \mu_{0}}{ }^{*} F_{\mu v} F^{\mu v},
\end{aligned}
$$

which yields: 


$$
\begin{aligned}
& \frac{d h_{m}}{d t}=-\frac{1}{2 c \mu_{0}} \int(V \mathbf{B}-\mathbf{A} \times \mathbf{E}) \cdot d \mathbf{S}-\frac{1}{c \mu_{0}} \int d^{3} r \mathbf{E} \cdot \mathbf{B}, \\
& \frac{d h_{e}}{d t}=-\frac{1}{2 c \mu_{0}} \int\left(V^{\prime} \mathbf{E}+\mathbf{C} \times \mathbf{B}\right) \cdot d \mathbf{S}+\frac{1}{c \mu_{0}} \int d^{3} r \mathbf{E} \cdot \mathbf{B} .
\end{aligned}
$$

In the special case that the domain of integration of Equation (17) is the whole $R^{3}$ space and the fields behave at infinity in a way such that the surface integrals in Equation (17) vanish, we get:

- If the integral of $\mathbf{E} \cdot \mathbf{B}$ is zero, both the magnetic and the electric helicities are constant during the evolution of the electromagnetic field.

- If the integral of $\mathbf{E} \cdot \mathbf{B}$ is not zero, the helicities are not constant, but they satisfy:

$$
\frac{d h_{m}}{d t}=-\frac{d h_{e}}{d t}
$$

so there is an interchange of helicities between the magnetic and electric parts of the field [8].

- For every value of the integral of $\mathbf{E} \cdot \mathbf{B}$, the electromagnetic helicity $h$, defined as:

$$
h=h_{m}+h_{e}=\frac{1}{2 c \mu_{0}} \int d^{3} r \mathbf{A} \cdot \mathbf{B}+\frac{\varepsilon_{0}}{2 c} \int d^{3} r \mathbf{C} \cdot \mathbf{E},
$$

is a conserved quantity.

If the domain of integration of Equation (17) is restricted to a finite volume $\Omega$, then the flux of electromagnetic helicity through the boundary $\partial \Omega$ of the volume is given by:

$$
\frac{d h}{d t}=-\frac{1}{2 c \mu_{0}} \int_{\partial \Omega}\left[(V \mathbf{B}-\mathbf{A} \times \mathbf{E})+\left(V^{\prime} \mathbf{E}+\mathbf{C} \times \mathbf{B}\right)\right] \cdot d \mathbf{S} .
$$

The integrand in the second term of this equation defines a vector density whose components are given by $\mathbf{S}_{i}=\mathcal{H}_{m}^{i}+\mathcal{H}_{e}^{i}$, so that:

$$
\mathbf{S}=\frac{1}{2 c^{2} \mu_{0}}\left(V \mathbf{B}-\mathbf{A} \times \mathbf{E}+V^{\prime} \mathbf{E}+\mathbf{C} \times \mathbf{B}\right) .
$$

This vector density has been considered as a physically meaningful spin density for the electromagnetic field in a vacuum in some references $[9,46,54-56]$. In the following, we examine some questions about the relation between the magnetic and electric parts of the helicity and their corresponding magnetic and electric parts of the spin.

\section{Fourier Decomposition and Helicity Basis for the Electromagnetic Field in a Vacuum}

In this section, we will write the electromagnetic fields in terms of the helicity basis, which will be very useful for obtaining the results and computations presented in the following sections.

The electric and magnetic fields can be decomposed into Fourier terms,

$$
\begin{aligned}
& \mathbf{E}(\mathbf{r}, t)=\frac{1}{(2 \pi)^{3 / 2}} \int d^{3} k\left(\mathbf{E}_{1}(\mathbf{k}) e^{-i k x}+\mathbf{E}_{2}(\mathbf{k}) e^{i k x)}\right), \\
& \mathbf{B}(\mathbf{r}, t)=\frac{1}{(2 \pi)^{3 / 2}} \int d^{3} k\left(\mathbf{B}_{1}(\mathbf{k}) e^{-i k x}+\mathbf{B}_{2}(\mathbf{k}) e^{i k x}\right),
\end{aligned}
$$

where we have introduced the four-dimensional notation $k x=\omega t-\mathbf{k} \cdot \mathbf{r}$, with $\omega=k c$. 
For the vector potentials, we need to fix a gauge. In the Coulomb gauge, the vector potentials are chosen so that $V=0, \nabla \cdot \mathbf{A}=0, V^{\prime}=0, \nabla \cdot \mathbf{C}=0$. Then, they satisfy the relations:

$$
\begin{gathered}
\mathbf{B}=\nabla \times \mathbf{A}=\frac{1}{c^{2}} \frac{\partial \mathbf{C}}{\partial t}, \\
\mathbf{E}=\nabla \times \mathbf{C}=-\frac{\partial \mathbf{A}}{\partial t} .
\end{gathered}
$$

One can write for them the following Fourier decomposition,

$$
\begin{aligned}
& \mathbf{A}(\mathbf{r}, t)=\frac{1}{(2 \pi)^{3 / 2}} \int d^{3} k\left[e^{-i k x} \overline{\mathbf{a}}(\mathbf{k})+e^{i k x} \mathbf{a}(\mathbf{k})\right], \\
& \mathbf{C}(\mathbf{r}, t)=\frac{c}{(2 \pi)^{3 / 2}} \int d^{3} k\left[e^{-i k x} \overline{\mathbf{c}}(\mathbf{k})+e^{i k x} \mathbf{c}(\mathbf{k})\right],
\end{aligned}
$$

where the factor $c$ in $\mathbf{C}$ is taken for dimensional reasons and $\overline{\mathbf{a}}, \overline{\mathbf{c}}$ denotes the complex conjugate of $\mathbf{a}, \mathbf{c}$, respectively. Taking time derivatives and using the Coulomb gauge conditions Equation (23),

$$
\begin{aligned}
& \mathbf{E}=-\frac{\partial \mathbf{A}}{\partial t}=\frac{1}{(2 \pi)^{3 / 2}} \int d^{3} k\left[e^{-i k x}(i k c) \overline{\mathbf{a}}(\mathbf{k})-e^{i k x}(i k c) \mathbf{a}(\mathbf{k})\right] \\
& \mathbf{B}=\frac{1}{c^{2}} \frac{\partial \mathbf{C}}{\partial t}=\frac{1}{(2 \pi)^{3 / 2}} \int d^{3} k\left[-e^{-i k x}(i k) \overline{\mathbf{c}}(\mathbf{k})+e^{i k x}(i k) \mathbf{c}(\mathbf{k})\right] .
\end{aligned}
$$

and by comparison with Equation (22), one can get the values for $\mathbf{a}(\mathbf{k})$ and $\mathbf{c}(\mathbf{k})$.

The helicity Fourier components appear when the vector potentials $\mathbf{A}$ and $\mathbf{C}$, in the Coulomb gauge, are written as a combination of circularly-polarized plane waves [57], as:

$$
\begin{aligned}
\mathbf{A}(\mathbf{r}, t) & =\frac{\sqrt{\hbar c \mu_{0}}}{(2 \pi)^{3 / 2}} \int \frac{d^{3} k}{\sqrt{2 k}}\left[e^{-i k x}\left(a_{R}(\mathbf{k}) \mathbf{e}_{R}(\mathbf{k})+a_{L}(\mathbf{k}) \mathbf{e}_{L}(\mathbf{k})\right)+C . C\right], \\
\mathbf{C}(\mathbf{r}, t) & =\frac{c \sqrt{\hbar c \mu_{0}}}{(2 \pi)^{3 / 2}} \int \frac{d^{3} k}{\sqrt{2 k}}\left[i e^{-i k x}\left(a_{R}(\mathbf{k}) \mathbf{e}_{R}(\mathbf{k})-a_{L}(\mathbf{k}) \mathbf{e}_{L}(\mathbf{k})\right)+C . C\right] .
\end{aligned}
$$

where $\hbar$ is the Planck constant and C.C means the complex conjugate. The Fourier components in the helicity basis are given by the unit vectors $\mathbf{e}_{R}(\mathbf{k}), \mathbf{e}_{L}(\mathbf{k}), \mathbf{e}_{k}=\mathbf{k} / k$, and the helicity components $a_{R}(\mathbf{k})$, $a_{L}(\mathbf{k})$ that, in the quantum theory, are interpreted as annihilation operators of photon states with rightand left-handed polarization, respectively. In quantum theory, $\bar{a}_{R}(\mathbf{k}), \bar{a}_{L}(\mathbf{k})$ are creation operators of such states.

In order to simplify the notation, most of the time, we will not write explicitly the dependence on $\mathbf{k}$ of the basis vectors and coefficients, meaning $a_{L}=a_{L}(\mathbf{k}), \mathbf{e}_{R}=\mathbf{e}_{R}(\mathbf{k}), a_{L}^{\prime}=a_{L}\left(\mathbf{k}^{\prime}\right), \mathbf{e}_{R}^{\prime}=\mathbf{e}_{R}\left(\mathbf{k}^{\prime}\right)$.

The unit vectors in the helicity basis are taken to satisfy:

$$
\begin{gathered}
\overline{\mathbf{e}}_{R}=\mathbf{e}_{L}, \mathbf{e}_{R}(-\mathbf{k})=-\mathbf{e}_{L}(\mathbf{k}), \mathbf{e}_{L}(-\mathbf{k})=-\mathbf{e}_{R}(\mathbf{k}), \\
\mathbf{e}_{k} \cdot \mathbf{e}_{R}=\mathbf{e}_{k} \cdot \mathbf{e}_{L}=0, \mathbf{e}_{R} \cdot \mathbf{e}_{R}=\mathbf{e}_{L} \cdot \mathbf{e}_{L}=0, \mathbf{e}_{R} \cdot \mathbf{e}_{L}=1, \\
\mathbf{e}_{k} \times \mathbf{e}_{k}=\mathbf{e}_{R} \times \mathbf{e}_{R}=\mathbf{e}_{L} \times \mathbf{e}_{L}=0, \\
\mathbf{e}_{k} \times \mathbf{e}_{R}=-i \mathbf{e}_{R}, \mathbf{e}_{k} \times \mathbf{e}_{L}=i \mathbf{e}_{L}, \mathbf{e}_{R} \times \mathbf{e}_{L}=-i \mathbf{e}_{k},
\end{gathered}
$$


The relation between the helicity basis and the planar Fourier basis can be obtained by comparing Equations (24) and (26). Consequently, the electric and magnetic fields of an electromagnetic field in a vacuum, and the vector potentials in the Coulomb gauge can be expressed in this basis as:

$$
\begin{aligned}
\mathbf{E}(\mathbf{r}, t) & =\frac{i c \sqrt{\hbar c \mu_{0}}}{(2 \pi)^{3 / 2}} \int d^{3} k \sqrt{\frac{k}{2}}\left[e^{-i k x}\left(a_{R} \mathbf{e}_{R}+a_{L} \mathbf{e}_{L}\right)-e^{i k x}\left(\bar{a}_{R} \mathbf{e}_{L}+\bar{a}_{L} \mathbf{e}_{R}\right)\right] \\
\mathbf{B}(\mathbf{r}, t) & =\frac{\sqrt{\hbar c \mu_{0}}}{(2 \pi)^{3 / 2}} \int d^{3} k \sqrt{\frac{k}{2}}\left[e^{-i k x}\left(a_{R} \mathbf{e}_{R}-a_{L} \mathbf{e}_{L}\right)+e^{i k x}\left(\bar{a}_{R} \mathbf{e}_{L}-\bar{a}_{L} \mathbf{e}_{R}\right)\right] \\
\mathbf{A}(\mathbf{r}, t) & =\frac{\sqrt{\hbar c \mu_{0}}}{(2 \pi)^{3 / 2}} \int d^{3} k \frac{1}{\sqrt{2 k}}\left[e^{-i k x}\left(a_{R} \mathbf{e}_{R}+a_{L} \mathbf{e}_{L}\right)+e^{i k x}\left(\bar{a}_{R} \mathbf{e}_{L}+\bar{a}_{L} \mathbf{e}_{R}\right)\right] \\
\mathbf{C}(\mathbf{r}, t) & =\frac{i c \sqrt{\hbar c \mu_{0}}}{(2 \pi)^{3 / 2}} \int d^{3} k \frac{1}{\sqrt{2 k}}\left[e^{-i k x}\left(a_{R} \mathbf{e}_{R}-a_{L} \mathbf{e}_{L}\right)-e^{i k x}\left(\bar{a}_{R} \mathbf{e}_{L}-\bar{a}_{L} \mathbf{e}_{R}\right)\right]
\end{aligned}
$$

where the unit vectors satisfy the relations Equation (27).

It is interesting to point the fact that in the helicity basis, we get for the magnetic vector potential the relation:

$$
\mathbf{A}(k)=-\frac{\mathbf{k} \times \mathbf{k} \times \mathbf{A}(k)}{\mathbf{k} \cdot \mathbf{k}},
$$

where:

$$
\mathbf{A}(k)=e^{-i k x}\left(a_{R} \mathbf{e}_{R}+a_{L} \mathbf{e}_{L}\right)+e^{i k x}\left(\bar{a}_{R} \mathbf{e}_{L}+\bar{a}_{L} \mathbf{e}_{R}\right),
$$

taken from Equation (28). In reference Equation [58], the nonlocality of electromagnetic quantities is discussed, and the transverse part of Fourier components of the vector potential is introduced as:

$$
\mathbf{A}^{\perp}(k)=-\frac{\mathbf{k} \times \mathbf{k} \times \mathbf{A}(k)}{\mathbf{k} \cdot \mathbf{k}} .
$$

We can see explicitly now from Equations (29) and (31) that in the Coulomb gauge in the helicity basis:

$$
\mathbf{A}(k)=\mathbf{A}^{\perp}(k) .
$$

\section{Magnetic and Electric Helicities in the Helicity Basis}

In the previous section, we have introduced the helicity basis and expressed the fields in that basis. In this section, we will express the electric and magnetic helicities in the same basis [7].

If we use the expressions (28), the magnetic helicity can be written as:

$$
\begin{aligned}
h_{m}= & \frac{1}{2 c \mu_{0}} \int d^{3} r \mathbf{A} \cdot \mathbf{B}=\frac{\hbar}{4} \int d^{3} k \int d^{3} k^{\prime} \int \frac{d^{3} r}{(2 \pi)^{3}} \sqrt{\frac{k^{\prime}}{k}} \\
& {\left[e^{-i \omega t} e^{i \omega^{\prime} t} e^{i\left(\mathbf{k}-\mathbf{k}^{\prime}\right) \cdot \mathbf{r}}\left(a_{R} \mathbf{e}_{R}+a_{L} \mathbf{e}_{L}\right) \cdot\left(\bar{a}_{R}^{\prime} \mathbf{e}_{L}^{\prime}-\bar{a}_{L}^{\prime} \mathbf{e}_{R}^{\prime}\right)\right.} \\
+ & e^{i \omega t} e^{-i \omega^{\prime} t} e^{-i\left(\mathbf{k}-\mathbf{k}^{\prime}\right) \cdot \mathbf{r}}\left(\bar{a}_{R} \mathbf{e}_{L}+\bar{a}_{L} \mathbf{e}_{R}\right) \cdot\left(a_{R}^{\prime} \mathbf{e}_{R}^{\prime}-a_{L}^{\prime} \mathbf{e}_{L}^{\prime}\right) \\
+ & e^{-i \omega t} e^{-i \omega^{\prime} t} e^{i\left(\mathbf{k}+\mathbf{k}^{\prime}\right) \cdot \mathbf{r}}\left(a_{R} \mathbf{e}_{R}+a_{L} \mathbf{e}_{L}\right) \cdot\left(a_{R}^{\prime} \mathbf{e}_{R}^{\prime}-a_{L}^{\prime} \mathbf{e}_{L}^{\prime}\right) \\
+ & \left.e^{i \omega t} e^{i \omega^{\prime} t} e^{-i\left(\mathbf{k}+\mathbf{k}^{\prime}\right) \cdot \mathbf{r}}\left(\bar{a}_{R} \mathbf{e}_{L}+\bar{a}_{L} \mathbf{e}_{R}\right) \cdot\left(\bar{a}_{R}^{\prime} \mathbf{e}_{L}^{\prime}-\bar{a}_{L}^{\prime} \mathbf{e}_{R}^{\prime}\right)\right] .
\end{aligned}
$$

Taking into account the following property of the Dirac-delta function,

$$
\int d^{3} k^{\prime} \int \frac{d^{3} r}{(2 \pi)^{3}} e^{-i\left(\mathbf{k}-\mathbf{k}^{\prime}\right) \cdot \mathbf{r}}\left(\mathbf{f}(\mathbf{k}) \cdot \mathbf{g}\left(\mathbf{k}^{\prime}\right)\right)=\mathbf{f}(\mathbf{k}) \cdot \mathbf{g}(\mathbf{k})
$$


and using the relations (27) yields:

$$
\begin{aligned}
h_{m} & =\frac{\hbar}{2} \int d^{3} k\left(\bar{a}_{R}(\mathbf{k}) a_{R}(\mathbf{k})-\bar{a}_{L}(\mathbf{k}) a_{L}(\mathbf{k})\right) \\
& +\frac{\hbar}{4} \int d^{3} k e^{-2 i \omega t}\left(-a_{R}(\mathbf{k}) a_{R}(-\mathbf{k})+a_{L}(\mathbf{k}) a_{L}(-\mathbf{k})\right) \\
& +\frac{\hbar}{4} \int d^{3} k e^{2 i \omega t}\left(-\bar{a}_{R}(\mathbf{k}) \bar{a}_{R}(-\mathbf{k})+\bar{a}_{L}(\mathbf{k}) \bar{a}_{L}(-\mathbf{k})\right) .
\end{aligned}
$$

We observe that the magnetic helicity has two contributions: the first term in Equation (34) is independent of time, and the rest of the terms constitute the time-dependent part of the magnetic helicity.

We repeat the same procedure for the electric helicity. The electric helicity can be written as:

$$
\begin{aligned}
h_{e}= & \frac{1}{2 c^{3} \mu_{0}} \int d^{3} r \mathbf{C} \cdot \mathbf{E}=\frac{\hbar}{4} \int d^{3} k \int d^{3} k^{\prime} \int \frac{d^{3} r}{(2 \pi)^{3}} \sqrt{\frac{k^{\prime}}{k}} \\
& {\left[e^{-i \omega t} e^{i \omega^{\prime} t} e^{i\left(\mathbf{k}-\mathbf{k}^{\prime}\right) \cdot \mathbf{r}}\left(a_{R} \mathbf{e}_{R}-a_{L} \mathbf{e}_{L}\right) \cdot\left(\bar{a}_{R}^{\prime} \mathbf{e}_{L}^{\prime}+\bar{a}_{L}^{\prime} \mathbf{e}_{R}^{\prime}\right)\right.} \\
+ & e^{i \omega t} e^{-i \omega^{\prime} t} e^{-i\left(\mathbf{k}-\mathbf{k}^{\prime}\right) \cdot \mathbf{r}}\left(\bar{a}_{R} \mathbf{e}_{L}-\bar{a}_{L} \mathbf{e}_{R}\right) \cdot\left(a_{R}^{\prime} \mathbf{e}_{R}^{\prime}+a_{L}^{\prime} \mathbf{e}_{L}^{\prime}\right) \\
- & e^{-i \omega t} e^{-i \omega^{\prime} t} e^{i\left(\mathbf{k}+\mathbf{k}^{\prime}\right) \cdot \mathbf{r}}\left(a_{R} \mathbf{e}_{R}-a_{L} \mathbf{e}_{L}\right) \cdot\left(a_{R}^{\prime} \mathbf{e}_{R}^{\prime}+a_{L}^{\prime} \mathbf{e}_{L}^{\prime}\right) \\
- & \left.e^{i \omega t} e^{i \omega^{\prime} t} e^{-i\left(\mathbf{k}+\mathbf{k}^{\prime}\right) \cdot \mathbf{r}}\left(\bar{a}_{R} \mathbf{e}_{L}-\bar{a}_{L} \mathbf{e}_{R}\right) \cdot\left(\bar{a}_{R}^{\prime} \mathbf{e}_{L}^{\prime}+\bar{a}_{L}^{\prime} \mathbf{e}_{R}^{\prime}\right)\right],
\end{aligned}
$$

and again using Equations (33) and ((27), we get,

$$
\begin{aligned}
h_{e} & =\frac{\hbar}{2} \int d^{3} k\left(\bar{a}_{R}(\mathbf{k}) a_{R}(\mathbf{k})-\bar{a}_{L}(\mathbf{k}) a_{L}(\mathbf{k})\right) \\
& -\frac{\hbar}{4} \int d^{3} k e^{-2 i \omega t}\left(-a_{R}(\mathbf{k}) a_{R}(-\mathbf{k})+a_{L}(\mathbf{k}) a_{L}(-\mathbf{k})\right) \\
& -\frac{\hbar}{4} \int d^{3} k e^{2 i \omega t}\left(-\bar{a}_{R}(\mathbf{k}) \bar{a}_{R}(-\mathbf{k})+\bar{a}_{L}(\mathbf{k}) \bar{a}_{L}(-\mathbf{k})\right) .
\end{aligned}
$$

The electromagnetic helicity $h$ in a vacuum is the sum of the magnetic and electric helicities. From Equations (34) and (36),

$$
h=h_{m}+h_{e}=\hbar \int d^{3} k\left(\bar{a}_{R}(\mathbf{k}) a_{R}(\mathbf{k})-\bar{a}_{L}(\mathbf{k}) a_{L}(\mathbf{k})\right) .
$$

In quantum electrodynamics, the integral in the right-hand side of Equation (37) is interpreted as the helicity operator, which subtracts the number of left-handed photons from the number of right-handed photons. From the usual expressions:

$$
\begin{aligned}
& N_{R}=\int d^{3} k \bar{a}_{R}(\mathbf{k}) a_{R}(\mathbf{k}), \\
& N_{L}=\int d^{3} k \bar{a}_{L}(\mathbf{k}) a_{L}(\mathbf{k})
\end{aligned}
$$

we can write (37) as:

$$
h=\hbar\left(N_{R}-N_{L}\right) .
$$

Consequently, the electromagnetic helicity (19) is the classical limit of the difference between the numbers of right-handed and left-handed photons $[6,7,15]$. 
However, the difference between the magnetic and electric helicities depends on time in general, since:

$$
\begin{aligned}
\tilde{h}(t)=h_{m}-h_{e}=\frac{\hbar}{2} \int d^{3} k & {\left[e^{-2 i \omega t}\left(-a_{R}(\mathbf{k}) a_{R}(-\mathbf{k})+a_{L}(\mathbf{k}) a_{L}(-\mathbf{k})\right)\right.} \\
+ & \left.e^{2 i \omega t}\left(-\bar{a}_{R}(\mathbf{k}) \bar{a}_{R}(-\mathbf{k})+\bar{a}_{L}(\mathbf{k}) \bar{a}_{L}(-\mathbf{k})\right)\right] .
\end{aligned}
$$

so the electromagnetic field is allowed to exchange electric and magnetic helicity components during its evolution. For an account of this phenomenon, we refer to $[8,25]$.

\section{Magnetic and Electric Spin in the Helicity Basis}

Now in this section, we are going to express the magnetic and electric spins components of the total angular momentum in the helicity basis.

Let us consider the spin vector defined by Equation (21). It can be written as:

$$
\mathbf{s}=\mathbf{s}_{m}+\mathbf{s}_{e},
$$

where the magnetic part of the spin is defined from the flux of magnetic helicity,

$$
\mathbf{s}_{m}=\frac{1}{2 c^{2} \mu_{0}} \int d^{3} r(V \mathbf{B}-\mathbf{A} \times \mathbf{E}),
$$

and the electric spin comes from the flux of the electric helicity,

$$
\mathbf{s}_{e}=\frac{1}{2 c^{2} \mu_{0}} \int d^{3} r\left(V^{\prime} \mathbf{E}+\mathbf{C} \times \mathbf{B}\right) .
$$

Note that the electric spin in Equation (43) can be defined only for the case of electromagnetism in a vacuum, in the same way as the electric helicity is defined only in a vacuum.

Using the helicity basis of the previous sections, which was calculated in the Coulomb gauge, the magnetic spin can be written as:

$$
\begin{aligned}
\mathbf{s}_{m}= & \frac{1}{2 c^{2} \mu_{0}} \int d^{3} r \mathbf{E} \times \mathbf{A}=\frac{\hbar}{4} \int d^{3} k \int d^{3} k^{\prime} \int \frac{d^{3} r}{(2 \pi)^{3}} \sqrt{\frac{k^{\prime}}{k}} \\
& {\left[i e^{-i \omega t} e^{i \omega^{\prime} t} e^{i\left(\mathbf{k}-\mathbf{k}^{\prime}\right) \cdot \mathbf{r}}\left(a_{R} \mathbf{e}_{R}+a_{L} \mathbf{e}_{L}\right) \times\left(\bar{a}_{R}^{\prime} \mathbf{e}_{L}^{\prime}+\bar{a}_{L}^{\prime} \mathbf{e}_{R}^{\prime}\right)\right.} \\
- & i e^{i \omega t} e^{-i \omega^{\prime} t} e^{-i\left(\mathbf{k}-\mathbf{k}^{\prime}\right) \cdot \mathbf{r}}\left(\bar{a}_{R} \mathbf{e}_{L}+\bar{a}_{L} \mathbf{e}_{R}\right) \times\left(a_{R}^{\prime} \mathbf{e}_{R}^{\prime}+a_{L}^{\prime} \mathbf{e}_{L}^{\prime}\right) \\
- & i e^{-i \omega t} e^{-i \omega^{\prime} t} e^{i\left(\mathbf{k}+\mathbf{k}^{\prime}\right) \cdot \mathbf{r}}\left(a_{R} \mathbf{e}_{R}+a_{L} \mathbf{e}_{L}\right) \times\left(a_{R}^{\prime} \mathbf{e}_{R}^{\prime}+a_{L}^{\prime} \mathbf{e}_{L}^{\prime}\right) \\
+ & \left.i e^{i \omega t} e^{i \omega^{\prime} t} e^{-i\left(\mathbf{k}+\mathbf{k}^{\prime}\right) \cdot \mathbf{r}}\left(\bar{a}_{R} \mathbf{e}_{L}+\bar{a}_{L} \mathbf{e}_{R}\right) \times\left(\bar{a}_{R}^{\prime} \mathbf{e}_{L}^{\prime}+\bar{a}_{L}^{\prime} \mathbf{e}_{R}^{\prime}\right)\right],
\end{aligned}
$$

and after the same manipulations as in the previous section, using Equations (33) and (27), it turns out:

$$
\begin{aligned}
\mathbf{s}_{m} & =\frac{\hbar}{2} \int d^{3} k\left(\bar{a}_{R}(\mathbf{k}) a_{R}(\mathbf{k})-\bar{a}_{L}(\mathbf{k}) a_{L}(\mathbf{k})\right) \mathbf{e}_{k} \\
& +\frac{\hbar}{4} \int d^{3} k e^{-2 i \omega t}\left(a_{R}(\mathbf{k}) a_{R}(-\mathbf{k})-a_{L}(\mathbf{k}) a_{L}(-\mathbf{k})\right) \mathbf{e}_{k} \\
& +\frac{\hbar}{4} \int d^{3} k e^{2 i \omega t}\left(\bar{a}_{R}(\mathbf{k}) \bar{a}_{R}(-\mathbf{k})-\bar{a}_{L}(\mathbf{k}) \bar{a}_{L}(-\mathbf{k})\right) \mathbf{e}_{k} .
\end{aligned}
$$

As in the case of magnetic helicity Equation (34), the magnetic spin has two contributions: the first term in Equation (45) is independent of time, while the rest of the terms are, in principle, time-dependent. 
In a similar way, the electric spin in the helicity basis is:

$$
\begin{aligned}
\mathbf{s}_{e}= & \frac{1}{2 c^{2} \mu_{0}} \int d^{3} r \mathbf{C} \times \mathbf{B}=\frac{\hbar}{4} \int d^{3} k \int d^{3} k^{\prime} \int \frac{d^{3} r}{(2 \pi)^{3}} \sqrt{\frac{k^{\prime}}{k}} \\
& {\left[i e^{-i \omega t} e^{i \omega^{\prime} t} e^{i\left(\mathbf{k}-\mathbf{k}^{\prime}\right) \cdot \mathbf{r}}\left(a_{R} \mathbf{e}_{R}-a_{L} \mathbf{e}_{L}\right) \times\left(\bar{a}_{R}^{\prime} \mathbf{e}_{L}^{\prime}-\bar{a}_{L}^{\prime} \mathbf{e}_{R}^{\prime}\right)\right.} \\
- & i e^{i \omega t} e^{-i \omega^{\prime} t} e^{-i\left(\mathbf{k}-\mathbf{k}^{\prime}\right) \cdot \mathbf{r}}\left(\bar{a}_{R} \mathbf{e}_{L}-\bar{a}_{L} \mathbf{e}_{R}\right) \times\left(a_{R}^{\prime} \mathbf{e}_{R}^{\prime}-a_{L}^{\prime} \mathbf{e}_{L}^{\prime}\right) \\
+ & i e^{-i \omega t} e^{-i \omega^{\prime} t} e^{i\left(\mathbf{k}+\mathbf{k}^{\prime}\right) \cdot \mathbf{r}}\left(a_{R} \mathbf{e}_{R}-a_{L} \mathbf{e}_{L}\right) \times\left(a_{R}^{\prime} \mathbf{e}_{R}^{\prime}-a_{L}^{\prime} \mathbf{e}_{L}^{\prime}\right) \\
- & \left.i e^{i \omega t} e^{i \omega^{\prime} t} e^{-i\left(\mathbf{k}+\mathbf{k}^{\prime}\right) \cdot \mathbf{r}}\left(\bar{a}_{R} \mathbf{e}_{L}-\bar{a}_{L} \mathbf{e}_{R}\right) \times\left(\bar{a}_{R}^{\prime} \mathbf{e}_{L}^{\prime}-\bar{a}_{L}^{\prime} \mathbf{e}_{R}^{\prime}\right)\right],
\end{aligned}
$$

that after integrating in $\mathbf{k}^{\prime}$ gives:

$$
\begin{aligned}
\mathbf{s}_{e} & =\frac{\hbar}{2} \int d^{3} k\left(\bar{a}_{R}(\mathbf{k}) a_{R}(\mathbf{k})-\bar{a}_{L}(\mathbf{k}) a_{L}(\mathbf{k})\right) \mathbf{e}_{k} \\
& +\frac{\hbar}{4} \int d^{3} k e^{-2 i \omega t}\left(-a_{R}(\mathbf{k}) a_{R}(-\mathbf{k})+a_{L}(\mathbf{k}) a_{L}(-\mathbf{k})\right) \mathbf{e}_{k} \\
& +\frac{\hbar}{4} \int d^{3} k e^{2 i \omega t}\left(-\bar{a}_{R}(\mathbf{k}) \bar{a}_{R}(-\mathbf{k})+\bar{a}_{L}(\mathbf{k}) \bar{a}_{L}(-\mathbf{k})\right) \mathbf{e}_{k} .
\end{aligned}
$$

Finally, the spin of the electromagnetic field in a vacuum is, according to Equation (41),

$$
\mathbf{s}=\mathbf{s}_{m}+\mathbf{s}_{e}=\hbar \int d^{3} k\left(\bar{a}_{R}(\mathbf{k}) a_{R}(\mathbf{k})-\bar{a}_{L}(\mathbf{k}) a_{L}(\mathbf{k})\right) \mathbf{e}_{k},
$$

an expression that is equivalent to the well-known result in quantum electrodynamics [57].

We can compute, as we did for the helicity, the difference between the magnetic and electric parts of the spin,

$$
\begin{aligned}
\tilde{\mathbf{s}}(t)=\mathbf{s}_{m}-\mathbf{s}_{e} & =\frac{\hbar}{2} \int d^{3} k\left[e^{-2 i \omega t}\left(a_{R}(\mathbf{k}) a_{R}(-\mathbf{k})-a_{L}(\mathbf{k}) a_{L}(-\mathbf{k})\right)\right. \\
& \left.+e^{2 i \omega t}\left(\bar{a}_{R}(\mathbf{k}) \bar{a}_{R}(-\mathbf{k})-\bar{a}_{L}(\mathbf{k}) \bar{a}_{L}(-\mathbf{k})\right)\right] \mathbf{e}_{k} .
\end{aligned}
$$

Note the similarity in the integrands of the difference between helicities Equation (40) and the difference between spins (49). Both have one term proportional to the complex quantity:

$$
f(\mathbf{k})=a_{R}(\mathbf{k}) a_{R}(-\mathbf{k})-a_{L}(\mathbf{k}) a_{L}(-\mathbf{k}),
$$

and another term proportional to the complex conjugate of $f(\mathbf{k})$. It is obvious that $f(\mathbf{k})$ is an even function of the wave vector $\mathbf{k}$. This means, in particular, that the integral Equation (49) is identically zero, so the spin difference satisfies:

$$
\tilde{\mathbf{s}}(t)=0 .
$$

Thus, we arrive at the following result for any electromagnetic field in a vacuum,

$$
\mathbf{s}_{m}=\mathbf{s}_{e}=\frac{1}{2} \mathbf{s}=\frac{\hbar}{2} \int d^{3} k\left(\bar{a}_{R}(\mathbf{k}) a_{R}(\mathbf{k})-\bar{a}_{L}(\mathbf{k}) a_{L}(\mathbf{k})\right) \mathbf{e}_{k} .
$$

This conclusion coincides with the results found in [46].

Therefore, while the magnetic and electric spins are equal in electromagnetism in a vacuum, in general, this fact does not apply to the magnetic and electric helicities, as we have seen in the previous section. These results have been obtained in the framework of standard classical electromagnetism in a vacuum, but they are also compatible with the suggestion made by Bliokh of a dual theory of electromagnetism [9]. 


\section{The Angular Momentum Decomposition for Non-Null Toroidal Electromagnetic Fields}

In this section, we calculate explicitly and analytically the spin-angular decomposition of a whole class of electromagnetic fields in a vacuum without using any paraxial approximation.

We will use the knotted non-null torus class [5,25]. These fields are exact solutions of Maxwell equations in a vacuum with the property that, at a given time $t=0$, all pairs of lines of the field $\mathbf{B}(\mathbf{r}, 0)$ are linked torus knots and that the linking number is the same for all the pairs. Similarly, for the electric field at the initial time $\mathbf{E}(\mathbf{r}, 0)$, all pairs of lines are linked torus knots, and the linking number is the same for all the pairs.

We take a four positive integers tuplet $(n, m, l, s)$. It is possible to find an initial magnetic field such that all its magnetic lines are $(n, m)$ torus knots. The linking number of every two magnetic lines at $t=0$ is equal to $n m$. Furthermore, we can find an initial electric field such that all the electric lines are $(l, s)$ torus knots and at $t=0$. At that time, the linking number of the electric field lines is equal to $l s$. We can assure that property at $t=0$, due to the fact that the topology may change during time evolution if one of the integers $(n, m, l, s)$ is different from any of the others (for details, we refer the interested reader to [5]). The magnetic and electric helicities also may change if the integer tuplet is not proportional to $(n, n, l, l)$. In these cases, the electromagnetic fields interchange the magnetic and electric helicities during their time evolution.

We define the dimensionless coordinates $(X, Y, Z, T)$, which are related to the physical ones $(x, y, z, t)$ by $(X, Y, Z, T)=(x, y, z, c t) / L_{0}$, and $r^{2} / L_{0}^{2}=\left(x^{2}+y^{2}+z^{2}\right) / L_{0}^{2}=X^{2}+Y^{2}+Z^{2}=R^{2}$. The length scale $L_{0}$ can be chosen to be the mean quadratic radius of the energy distribution of the electromagnetic field. The set of non-null torus electromagnetic knots can be written as:

$$
\begin{aligned}
\mathbf{B}(\mathbf{r}, t) & =\frac{\sqrt{a}}{\pi L_{0}^{2}} \frac{Q \mathbf{H}_{1}+P \mathbf{H}_{2}}{\left(A^{2}+T^{2}\right)^{3}} \\
\mathbf{E}(\mathbf{r}, t) & =\frac{\sqrt{a} c}{\pi L_{0}^{2}} \frac{Q \mathbf{H}_{4}-P \mathbf{H}_{3}}{\left(A^{2}+T^{2}\right)^{3}}
\end{aligned}
$$

where $a$ is a constant related to the energy of the electromagnetic field,

$$
A=\frac{1+R^{2}-T^{2}}{2}, P=T\left(T^{2}-3 A^{2}\right), Q=A\left(A^{2}-3 T^{2}\right)
$$

and:

$$
\begin{gathered}
\mathbf{H}_{1}=(-n X Z+m Y+s T) \mathbf{u}_{x}+(-n Y Z-m X-l T Z) \mathbf{u}_{y} \\
+\left(n \frac{-1-Z^{2}+X^{2}+Y^{2}+T^{2}}{2}+l T Y\right) \mathbf{u}_{z} . \\
\mathbf{H}_{2}=\left(s \frac{1+X^{2}-Y^{2}-Z^{2}-T^{2}}{2}-m T Y\right) \mathbf{u}_{x}+(s X Y-l Z+m T X) \mathbf{u}_{y}+(s X Z+l Y+n T) \mathbf{u}_{z} . \\
\mathbf{H}_{3}=(-m X Z+n Y+l T) \mathbf{u}_{x}+(-m Y Z-n X-s T Z) \mathbf{u}_{y} \\
+\left(m \frac{-1-Z^{2}+X^{2}+Y^{2}+T^{2}}{2}+s T Y\right) \mathbf{u}_{z} . \\
\mathbf{H}_{4}=\left(l \frac{1+X^{2}-Y^{2}-Z^{2}-T^{2}}{2}-n T Y\right) \mathbf{u}_{x}+(l X Y-s Z+n T X) \mathbf{u}_{y}+(l X Z+s Y+m T) \mathbf{u}_{z} .
\end{gathered}
$$

The energy $\mathcal{E}$, linear momentum $\mathbf{p}$ and total angular momentum $\mathbf{J}$ of these fields are:

$$
\begin{aligned}
\mathcal{E} & =\int\left(\frac{\varepsilon_{0} E^{2}}{2}+\frac{B^{2}}{2 \mu_{0}}\right) d^{3} r=\frac{a}{2 \mu_{0} L_{0}}\left(n^{2}+m^{2}+l^{2}+s^{2}\right) \\
\mathbf{p} & =\int \varepsilon_{0} \mathbf{E} \times \mathbf{B} d^{3} r=\frac{a}{2 c \mu_{0} L_{0}}(l n+m s) \mathbf{u}_{y} \\
\mathbf{J} & =\int \varepsilon_{0} \mathbf{r} \times(\mathbf{E} \times \mathbf{B}) d^{3} r=\frac{a}{2 c \mu_{0}}(l m+n s) \mathbf{u}_{y}
\end{aligned}
$$


To study the interchange between the magnetic and electric helicities and the spins, we first need the Fourier transforms of the fields in the helicity basis. Following the prescription given in Section 3, we get:

$$
\begin{aligned}
a_{R} \mathbf{e}_{R}+a_{L} \mathbf{e}_{L} & =\sqrt{\frac{a}{\hbar c \mu_{0}}} \frac{L_{0}^{3 / 2}}{2 \sqrt{\pi}} \frac{e^{-K}}{\sqrt{K}} \times\left[\frac{m}{K}\left(K_{x} K_{z}, K_{y} K_{z},-K_{x}^{2}-K_{y}^{2}\right)+s\left(0, K_{z},-K_{y}\right)\right] \\
& +i\left[\frac{l}{K}\left(-K_{y}^{2}-K_{z}^{2}, K_{x} K_{y}, K_{x} K_{z}\right)+n\left(-K_{y}, K_{x}, 0\right)\right] \\
a_{R} \mathbf{e}_{R}-a_{L} \mathbf{e}_{L} & =\sqrt{\frac{a}{\hbar c \mu_{0}}} \frac{L_{0}^{3 / 2}}{2 \sqrt{\pi}} \frac{e^{-K}}{\sqrt{K}} \times\left[\frac{n}{K}\left(K_{x} K_{z}, K_{y} K_{z},-K_{x}^{2}-K_{y}^{2}\right)+l\left(0, K_{z},-K_{y}\right)\right] \\
& +i\left[\frac{s}{K}\left(-K_{y}^{2}-K_{z}^{2}, K_{x} K_{y}, K_{x} K_{z}\right)+m\left(-K_{y}, K_{x}, 0\right)\right]
\end{aligned}
$$

In these expressions, we have introduced the dimensionless Fourier space coordinates $\left(K_{x}, K_{y}, K_{z}\right)$, related to the dimensional Fourier space coordinates $\left(k_{x}, k_{y}, k_{z}\right)$ according to:

$$
\left(K_{x}, K_{y}, K_{z}\right)=L_{0}\left(k_{x}, k_{y}, k_{z}\right), \quad K=L_{0} k=\frac{L_{0} \omega}{c} .
$$

The electromagnetic helicity Equation (37) of the set of non-null torus electromagnetic knots results:

$$
h=\hbar \int d^{3} k\left(\bar{a}_{R}(\mathbf{k}) a_{R}(\mathbf{k})-\bar{a}_{L}(\mathbf{k}) a_{L}(\mathbf{k})\right)=\frac{a}{2 c \mu_{0}}(n m+l s),
$$

and the difference between the magnetic and electric helicities is:

$$
\begin{aligned}
\tilde{h}(t) & =h_{m}-h_{e}=\frac{\hbar}{2} \int d^{3} k\left[e^{-2 i \omega t}\left(-a_{R}(\mathbf{k}) a_{R}(-\mathbf{k})+a_{L}(\mathbf{k}) a_{L}(-\mathbf{k})\right)\right. \\
& \left.+e^{2 i \omega t}\left(-\bar{a}_{R}(\mathbf{k}) \bar{a}_{R}(-\mathbf{k})+\bar{a}_{L}(\mathbf{k}) \bar{a}_{L}(-\mathbf{k})\right)\right] \\
& =\frac{a}{2 c \mu_{0}}(n m-l s) \frac{1-6 T^{2}+T^{4}}{\left(1+T^{2}\right)^{4}}
\end{aligned}
$$

where we recall that $T=c t / L_{0}$. Results Equations (66) and (67) coincide with the computations done in [5] using different procedures.

Now, consider the spin in Equation (48). For the set of non-null torus electromagnetic knots, we get:

$$
\mathbf{s}=\hbar \int d^{3} k\left(\bar{a}_{R}(\mathbf{k}) a_{R}(\mathbf{k})-\bar{a}_{L}(\mathbf{k}) a_{L}(\mathbf{k})\right) \mathbf{e}_{k}=\frac{a}{4 c \mu_{0}}(m l+n s) \mathbf{u}_{y} .
$$

Notice that this value of spin is equal to one half of the value of the total angular momentum obtained in Equation (62). Thus, the orbital angular momentum of this set of electromagnetic fields has the same value as the spin angular momentum,

$$
\mathbf{L}=\mathbf{s}=\frac{1}{2} \mathbf{J} .
$$

The difference between the magnetic and the electric spin can also be computed through Equation (49). The result is:

$$
\begin{aligned}
\tilde{\mathbf{s}}(t) & =\mathbf{s}_{m}-\mathbf{s}_{e}=\frac{\hbar}{2} \int d^{3} k\left[e^{-2 i \omega t}\left(a_{R}(\mathbf{k}) a_{R}(-\mathbf{k})-a_{L}(\mathbf{k}) a_{L}(-\mathbf{k})\right)\right. \\
& \left.+e^{2 i \omega t}\left(\bar{a}_{R}(\mathbf{k}) \bar{a}_{R}(-\mathbf{k})-\bar{a}_{L}(\mathbf{k}) \bar{a}_{L}(-\mathbf{k})\right)\right] \mathbf{e}_{k}=\mathbf{0} .
\end{aligned}
$$


As a consequence, even if the magnetic and electric helicities depend on time for this set of electromagnetic fields, the magnetic and electric parts of the spin are time independent, satisfying the results found in Equation (52) for general electromagnetic fields in a vacuum. Both are equal, and satisfy:

$$
\mathbf{s}_{m}=\mathbf{s}_{e}=\frac{1}{2} \mathbf{s}=\frac{a}{8 c \mu_{0}}(m l+n s) \mathbf{u}_{y} .
$$

\section{Same Spin-Orbital Decomposition with Different Behavior in the Helicities}

In this section, we consider two knotted electromagnetic fields in which the spin and orbital decomposition of the angular momentum are equal in both cases, while the helicities are constant and non-constant, respectively. We will see that the angular momentum density evolves differently in each case.

In the first case, we take the set $(n, m, l, s)=(5,3,5,3)$ in Equations (53) and (54). Thus, using Equation (62) the total angular momentum is:

$$
\mathbf{J}=\frac{15 a}{\mu_{0}} \mathbf{u}_{y}
$$

while the angular density changes in time. In order to visualize the evolution of the angular momentum density, which is given by $\mathbf{j}=\mathbf{r} \times(\mathbf{E} \times \mathbf{B})$, we plot at different times the vector field sample at the plane $X Z$, as is depicted in Figure 1.
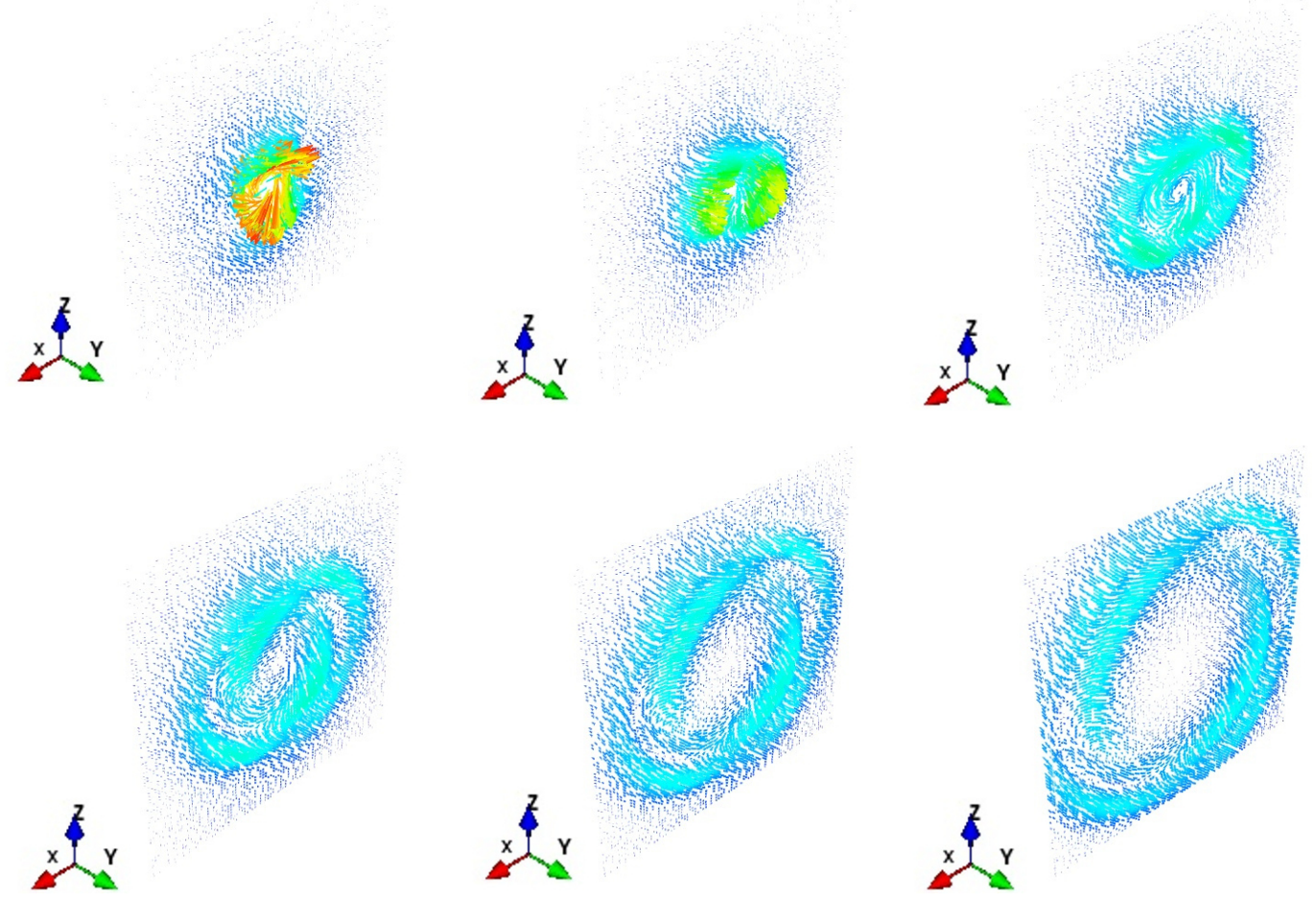

Figure 1. The angular momentum density $\mathbf{j}$ at times $T=0,0.5,1,1.5,2,2.5$, for the electromagnetic field given by the set $(n, m, l, s)=(5,3,5,3)$. The vector field is sampled at the plane XZ. In the case depicted in the figure, the magnetic helicity is equal to the electric helicity and constant in time.

For this case, the spin-orbital split, as shown in the previous section, using Equation (69), turns out to be:

$$
\mathbf{L}=\mathbf{s}=\frac{15 a}{2 \mu_{0}} \mathbf{u}_{y}
$$


which remains constant during the time evolution of the field. The magnetic and electric helicities remain also constant, and there is no exchange between them.

Now, let us take the set $(n, m, l, s)=(15,5,0,2)$ in Equations (53) and (54). The electromagnetic field obtained with this set of integers has the same value of the total angular momentum as the previous case and the same spin-orbital split. However, in this case, the magnetic and electric helicities are time-dependent, satisfying Equation (67). The time evolution of the angular momentum density is different from the case of constant helicities, as we can see in Figure 2. As we did before, we have plotted the field at the plane $\mathrm{XZ}$ at the same time steps as in the first example.
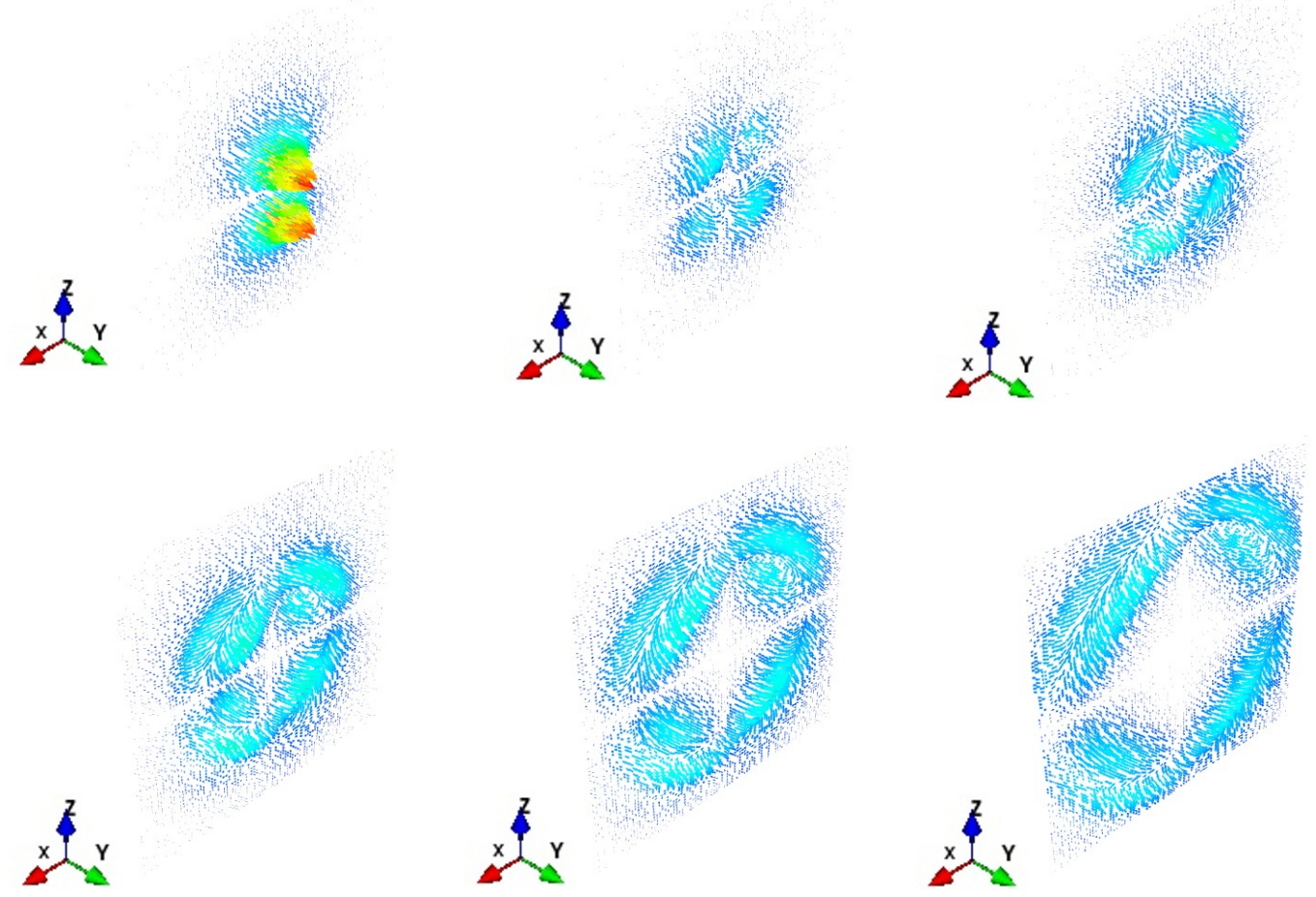

Figure 2. The angular momentum density $\mathbf{j}$ at times $T=0,0.5,1,1.5,2,2.5$, for the electromagnetic field given by the set $(n, m, l, s)=(15,5,0,2)$. The vector field is sampled at the plane $X Z$. In this example, the magnetic and electric helicities are initially different, and their values change with time.

In the first example of a non-null torus electromagnetic field, the helicities remain constant in time. In the second example, the magnetic helicity is initially different from the electric helicity, and both change with time. Even if the spin, orbital and total angular momenta are equal in both examples, we can see in Figures 1 and 2 that the structure of the total angular momentum density is different. We can speculate that a macroscopic particle, which can interact with the angular momentum of the field, would behave in the same way in both cases, but a microscopic test particle able to interact with the local density of the angular momentum would behave differently.

\section{Conclusions}

We have calculated analytically and exactly the spin-orbital decomposition of the angular momentum of a class of electromagnetic fields beyond the paraxial approximation. A spin density that is dual in its magnetic and electric contributions has been considered. This spin density has the meaning of flux of electromagnetic helicity. By using a Fourier decomposition of the electromagnetic field in a vacuum in terms of circularly-polarized waves, called the helicity basis, we have given explicit expressions for the magnetic and electric contributions to the spin angular momentum. We have obtained the results that the magnetic and electrical components of spin remain constant during the 
time evolution of the fields. We also have made use of the helicity basis to calculate the magnetic and electric helicities.

We have obtained the exact split of the angular momentum into spin and orbital components for electromagnetic fields, which belong to the non-null toroidal knotted class [5]. One of main characteristics of that class is that it contains a certain degree of linkage of electric and magnetic lines and can have exchange between the magnetic and electrical components of the helicity [8].

We have considered two examples of these non-null knotted electromagnetic fields having the properties that they have the same angular momentum and the same split. They have the same constant values for the orbital and spin components of the angular momentum, the first with constant and equal helicities and the second with time-evolving helicities. The behavior of the total angular momentum density seems to be different in these two cases.

In our opinion, the study of this kind of example with nontrivial helicities may provide a clarification of the role of helicities in the behavior of angular momentum densities of electromagnetic fields in a vacuum.

Acknowledgments: We acknowledge Wolfgang Löffler for valuable discussions. This work was supported by research grants from the Spanish Ministry of Economy and Competitiveness (MINECO/FEDER) ESP2015-69909-C5-4-R.

Author Contributions: Manuel Arrayás and José L. Trueba conceived of all the results of this work, made the computations and wrote the paper.

Conflicts of Interest: The authors declare no conflict of interest. The founding sponsors had no role in the design of the study; in the collection, analyses or interpretation of data; in the writing of the manuscript; nor in the decision to publish the results.

\section{References}

1. Van Enk, S.J.; Nienhuis, G. Spin and orbital angular momentum of photons. Europhys. Lett. 1994, 25, 497-501.

2. Van Enk, S.J;; Nienhuis, G. Commutation rules and eigenvalues of spin and orbital angular momentum of radiation fields. J. Mod. Opt. 1994, 41, 963-977.

3. Allen, L.; Barnett, S.M.; Padgett, M.J. (Eds.) Optical Angular Momentum; Institute of Physics: Bristol, UK, 2003.

4. Bliokh, K.Y.; Aiello, A.; Alonso, M. The Angular Momentum of Light; Andrews, D.L., Babiker, M., Eds; Cambridge University Press: Hong Kong, China, 2012.

5. Arrayás, M.; Trueba, J.L. A class of non-null toroidal electromagnetic fields and its relation to the model of electromagnetic knots. J. Phys. A Math. Theor. 2015, 48, 025203.

6. Afanasiev, G.N.; Stepanovsky, Y.P. The helicity of the free electromagnetic field and its physical meaning. Nuovo Cim. A 1996, 109, 271-279.

7. Trueba, J.L.; Rañada, A.F. The electromagnetic helicity. Eur. J. Phys. 1996, 17, 141-144.

8. Arrayás, M.; Trueba, J.L. Exchange of helicity in a knotted electromagnetic field. Ann. Phys. (Berl.) 2012, 524, $71-75$.

9. Bliokh, K.Y.; Bekshaev, A.Y.; Nori, F. Dual electromagnetism: Helicity, spin, momentum and angular momentum. New J. Phys. 2013, 15, 033026.

10. Berry, M.V. Optical currents. J. Opt. A Pure Appl. Opt. 2009, 11, 094001.

11. Ra nada, A.F. A topological theory of the electromagnetic field. Lett. Math. Phys. 1989, 18, 97-106.

12. Ra nada, A.F. Knotted solutions of the Maxwell equations in a vacuum. J. Phys. A Math. Gen. 1990, 23, L815-L820.

13. Ra nada, A.F. Topological electromagnetism. J. Phys. A Math. Gen. 1992, 25, 1621-1641.

14. Ra nada, A.F.; Trueba, J.L. Electromagnetic knots. Phys. Lett. A 1995, 202, 337-342.

15. Ra nada, A.F.; Trueba, J.L. Two properties of electromagnetic knots. Phys. Lett. A 1997, 232, 25-33.

16. Ra nada, A.F.; Trueba, J.L. A topological mechanism of discretization for the electric charge. Phys. Lett. B 1998, 422, 196-200.

17. Ra nada, A.F.; Trueba, J.L. Topological Electromagnetism with Hidden Nonlinearity. In Modern Nonlinear Optics III; Evans, M.W., Ed.; John Wiley \& Sons: New York, NY, USA, 2001; pp 197-253.

18. Irvine, W.T.M.; Bouwmeester, D. Linked and knotted beams of light. Nat. Phys. 2008, 4, 716-720. 
19. Besieris, I.M.; Shaarawi, A.M. Hopf-Rañada linked and knotted light beam solution viewed as a null electromagnetic field. Opt. Lett. 2009, 34, 3887-3889.

20. Arrayás, M.; Trueba, J.L. Motion of charged particles in a knotted electromagnetic field. J. Phys. A Math. Theor. 2010, 43, 235401.

21. Van Enk, S.J. The covariant description of electric and magnetic field lines of null fields: Application to Hopf-Rañada solutions. J. Phys. A Math. Theor. 2013, 46, 175204.

22. Kedia, H.; Bialynicki-Birula, I.; Peralta-Salas, D.; Irvine, W.T.M. Tying knots in light fields. Phys. Rev. Lett. 2013, 111, 150404.

23. Hoyos, C.; Sircar, N.; Sonnenschein, J. New knotted solutions of Maxwell's equations. J. Phys. A Math. Theor. 2015, 48, 255204.

24. Kedia, H.; Foster, D.; Dennis, M.R.; Irvine, W.T.M. Weaving knotted vector fields with tunable helicity. Phys. Rev. Lett. 2016, 117, 274501.

25. Arrayás, M.; Bouwmeester, D.; Trueba, J.L. Knots in electromagnetism. Phys. Rep. 2017, 667, 1-61.

26. Arrayás, M.; Trueba, J.L. Collision of two hopfions. J. Phys. A Math. Theor. 2017, 50, 085203.

27. Kamchatnov, A.M. Topological solitons in magnetohydrodynamics. Zh. Eksp. Teor. Fiz. 1982, 82, 117-124.

28. Semenov, V.S.; Korovinski, D.B.; Biernat, H.K. Euler potentials for the MHD Kamchatnov-Hopf soliton solution. Nonlinear Process. Geophys. 2002, 9, 347-354.

29. Thompson, A.; Swearngin, J.; Wickes, A.; Bouwmeester, D. Constructing a class of topological solitons in magnetohydrodynamics. Phys. Rev. E 2014, 89, 043104.

30. Smiet, C.B.; Candelaresi, S.; Thompson, A.; Swearngin, J.; Dalhuisen, J.W.; Bouwmeester, D. Self-organizing knotted magnetic structures in plasma. Phys. Rev. Lett. 2015, 115, 095001.

31. O'Holleran, K.; Dennis, M.R.; Padgett, M.J. Topology of light's darkness. Phys. Rev. Lett. 2009, 102, 143902.

32. Dennis, M.R.; King, R.P.; Jack, B.; O’Holleran, K.; Padgett, M.J. Isolated optical vortex knots. Nat. Phys. 2010, 6, 118-121.

33. Romero, J.; Leach, J.; Jack, B.; Dennis, M.R.; Franke-Arnold, S.; Barnett, S.M.; Padgett, M.J. Entangled optical vortex links. Phys. Rev. Lett. 2011, 106, 100407.

34. Desyatnikov, A.S.; Buccoliero, D.; Dennis, M.R.; Kivshar, Y.S. Spontaneous knotting of self-trapped waves. Sci. Rep. 2012, 2, 771 .

35. Rubinsztein-Dunlop, H.; Forbes, A.; Berry, M.V.; Dennis, M.R.; Andrews, D.L.; Mansuripur, M.; Denz, C.; Alpmann, C.; Banzer, P.; Bauer, T.; et al. Roadmap on Structured Light. J. Opt. 2017, 19, 013001.

36. Faddeev, L.; Niemi, A.J. Stable knot-like structures in classical field theory. Nature 1997, 387, 58-61.

37. Hall, D.S.; Ray, M.W.; Tiurev, K.; Ruokokoski, E.; Gheorge, A.H.; Möttönen, M. Tying quantum knots. Nat. Phys. 2016, 12, 478-483.

38. Taylor, A.J.; Dennis, M.R. Vortex knots in tangled quantum eigenfunctions. Nat. Commun. 2016, 7, 12346.

39. Volovik, G.E.; Mineev, V.O. Particle-like solitons in superfluid He phases. Zh. Eksp. Teor. Fiz. 1977, 73, 767-773.

40. Dzyloshinskii, I.; Ivanov, B. Localized topological solitons in a ferromagnet. Pis'ma Zh. Eksp. Teor. Fiz. 1979, 29, 592-595.

41. Kawaguchi, Y.; Nitta, M.; Ueda, M. Knots in a spinor Bose-Einstein condensate. Phys. Rev. Lett. 2008, 100, 180403.

42. Kleckner, A.; Irvine, W.T.M. Creation and dynamics of knotted vortices. Nat. Phys. 2013, 9, 253-258.

43. Kleckner, A.; Irvine, W.T.M. Liquid crystals: Tangled loops and knots. Nat. Mat. 2014, 13, 229-231.

44. Dalhuisen, J.W.; Bouwmeester, D. Twistors and electromagnetic knots. J. Phys. A Math. Theor. 2012, 45, 135201.

45. Thompson, A.; Swearngin, J.; Wickes, A.; Bouwmeester, D. Classification of electromagnetic and gravitational hopfions by algebraic type. J. Phys. A Math. Theor. 2015, 48, 205202.

46. Barnett, S.M. On the six components of optical angular momentum. J. Opt. 2011, 13, 064010.

47. Stratton, J.A. Electromagnetic Theory; McGraw-Hill: New York, NY, USA, 1941.

48. Moffatt, H.K. The degree of knottedness of tangled vortex lines. J. Fluid Mech. 1969, 35, 117-129.

49. Berger, M.A.; Field, G.B. The topological properties of magnetic helicity. J. Fluid Mech. 1984, 147, $133-148$.

50. Moffatt, H.K.; Ricca, R.L. Helicity and the Calugareanu Invariant. Proc. R. Soc. A 1992, 439, 411-429.

51. Berger, M.A. Introduction to magnetic helicity. Plasma Phys. Control. Fusion 1999, 41, B167-B175.

52. Dennis, M.R.; Hannay, J.H. Geometry of Calugareanu's theorem. Proc. R. Soc. A 2005, 461, 3245-3254.

53. Ricca, R.L.; Nipoti, B. Gauss' linking number revisited. J. Knot Theor. Ramif. 2011, 20, 1325-1343.

54. Bliokh, K.Y.; Alonso, M.A.; Ostrovskaya, E.A.; Aiello, A. Angular momenta and spin-orbit interaction of nonparaxial light in free space. Phys. Rev. A 2010, 82, 063825. 
55. Barnett, S.M. Rotation of electromagnetic fields and the nature of optical angular momentum. J. Mod. Opt. 2010, 57, 1339-1343.

56. Bialynicki-Birula, I.; Bialynicki-Birula, Z. Canonical separation of angular momentum of light into its orbital and spin parts. J. Opt. 2011, 13, 064014.

57. Ynduráin, F.J. Mecánica Cuántica; Alianza Editorial: Madrid, Spain, 1988.

58. Bialynicki-Birula, I. Local and nonlocal observables in quantum optics. New J. Phys. 2014, 16, 113056.

(C) 2018 by the authors. Licensee MDPI, Basel, Switzerland. This article is an open access article distributed under the terms and conditions of the Creative Commons Attribution (CC BY) license (http:/ / creativecommons.org/licenses/by/4.0/). 